(C) 2014 IEEE. Personal use of this material is permitted. Permission from IEEE must be obtained for all other uses, in any current or future media, including reprinting/republishing this material for advertising or promotional purposes, creating new collective works, for resale or redistribution to servers or lists, or reuse of any copyrighted component of this work in other works. 


\section{Error Exponent of Amplify and Forward Relay Networks in Presence of I.I.D. Interferers}

\author{
Bappi Barua and Farzad Safaei \\ ICT Research Institute \\ Faculty of Informatics \\ University of Wollongong \\ Wollongong, NSW 2522, Australia \\ email: b.barua@ieee.org,farzad@uow.edu.au
}

\author{
Mehran Abolhasan \\ Faculty of Engineering and IT \\ University of Technology Sydney \\ Sydney, NSW 2007, Australia \\ email: mehran.abolhasan@uts.edu.au
}

\begin{abstract}
In this paper, we derive the random coding error exponent of amplify-and-forward (AF) relay networks in presence of arbitrary number of independent and identically distributed (i.i.d.) interferers both at the relay and the destination. Multiuser networks are common examples of interference limited networks. We derive the ergodic capacity of the network and present simulation results on the performance of the network where we compare the capacity and error exponent performance of interference limited networks with noise limited networks. Numerical results show that noise limited networks outperform interference limited networks even when only a very few interferers exist in the network.
\end{abstract}

Index Terms-Interference network, Random coding error exponent, amplify-and-forward, ergodic capacity

\section{INTRODUCTION}

Capacity analysis of wireless networks is crucial to determine the reliable data rate that a channel can provide. Capacity bounds of general relay channel has been studied in many papers using information theory [1]-[3]. However, most of these have characterized capacity bounds for fading and Gaussian relay networks. An approximate capacity bound has been derived for a Gaussian interference relay network in [3]. In reality, with the growing number of wireless devices interference is becoming unavoidable in practical networks. Performance analysis of interference in cooperative relay networks has been studied extensively in [4]-[7]. The authors in [4], [5] consider a scenario where the relay node is affected by interference in an interference limited network and the receiver node remains interference free. The relays considered can estimate the instantaneous channel state information (CSI) of interfering channels to use it in the gain. However, the assumption of AF relay gain parameter that includes the instantaneous or average channel information of interfering channels as considered in [4]-[6] requires additional computational capability at the relaying node, and in certain cases where the interfering signals are not known to the relay a priori, the technique is not applicable. A total interference limited cooperative network has been studied in [6], [7]. Outage performance of a dual hop network has been investigated using a fixed gain relay in [6] and hypothetical gain AF relay in [7] with arbitrary number of interferes.
C. E. Shannon defined a reliability function or error exponent to describe the probability of the error as a function of code rate $R$ and code length $W$ as,

$$
E(R) \triangleq \lim _{W \rightarrow \infty} \sup \frac{-\ln P_{e}^{\text {opt }}(R, W)}{W}
$$

where $P_{e}^{\text {opt }}(R, W)$ is the average block error probability for the optimal block code of length $W$ and rate $R$ [8]. In practice, derivation of exact error exponent (1) involves quite complex mathematical procedures, however, a lower bound on the error exponent known as random coding error exponent (RCEE), (defined in [9], [10]) exists. This RCEE measurement provides important information about the design requirements of a codeword to achieve a given target rate $R$ below the capacity $C$ of the channel. In [11] and [12], the authors have derived the RCEE of cooperative relay networks and two way relay networks respectively using CSI assisted ideal gain AF relays and obtained the ergodic capacity and cutoff rate for the network. Recently, the random coding error exponent and the capacity of a dual hop cooperative relay network using single antenna CSI assisted AF relay were derived in [13]. However, all the analysis regarding RCEE has been performed for noise limited relay networks only, and to the best of our knowledge RCEE and capacity analysis of cooperative relay networks in interference using RCEE has not been studied to date.

In this paper, we derive the closed form RCEE of cooperative relay network in presence of arbitrary number of i.i.d. interferers using an ideal gain $\mathrm{AF}$ relay. I.i.d. interferers can represent the worst case scenario of interference limited networks in a similar interference power constraints. In [14], the authors show that the performance of interference network does not depend on individual interferer's power but on the aggregated power of the interferers. Thus, our i.i.d. assumption will provide a lower bound on the performance of the nonidentically distributed interference network, considering that the maximum power of the nonidentically distributed interferer is allocated to the i.i.d interferers. Furthermore, we provide the ergodic capacity expression of the network. 


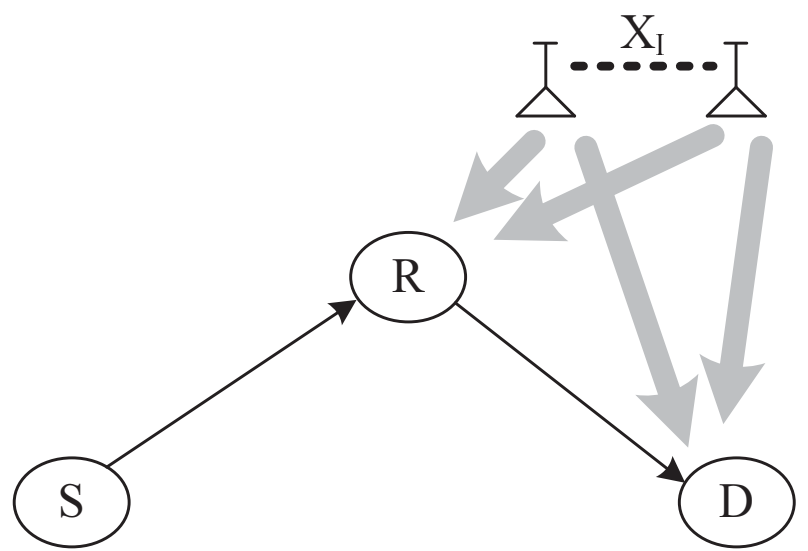

Fig. 1. System model of interference relay network where interferers are both at the relay and the destination.

\section{System Model}

Consider a single source-destination pair communicating via a single antenna amplify-and-forward relay without any direct link. We will denote source-relay and relay-destination links as $\mathrm{S}-\mathrm{R}$ and R-D respectively. We assume the main channels (S-R and R-D) and all the interfering channels are Rayleigh faded. Furthermore, the destination is assumed to have full channel state information (CSI) of the two main channels, while the relay has full CSI of the S-R channel only. The source and relay have no CSI of forwarding transmitting channels. None of the nodes, source (S), relay (R) and destination (D) possess information about the interfering channels.

The instantaneous and average signal power of 1 st and 2 nd hops are denoted as $\gamma_{i} \triangleq P\left|h_{i}\right|^{2}$ and $\lambda_{i} \triangleq P \Omega_{i}$ respectively, where $i \in\{1,2\}, P$ is the corresponding source and relay power; $h_{i}$ and $\Omega_{i}$ are the instantaneous and average channel gain of the $i$ th hop respectively. The average channel gain, $\Omega_{i}$ is in fact the statistical average of the squared instantaneous channel gain $h_{i}$, i.e. $\Omega_{i} \triangleq \mathbb{E}\left[\left|h_{i}\right|^{2}\right]$.

Consider a total of $L$ interferers in the system, then the instantaneous and the average interference power of any interferer $I_{l}$ is $\gamma_{I, l} \triangleq P_{I, l}\left|h_{I, l}\right|^{2}$ and $\lambda_{I, l} \triangleq P_{I, l} \Omega_{I, l}$ respectively. $h_{I, l}$ is the fading channel gain from the interfering source $I_{l}$ to the node $i$ respectively, $l \in\{1,2, \ldots L\}$ and $i \in\{R, D\} . \Omega_{I, l}$ is the average interfering channel gain, $\Omega_{I, l} \triangleq \mathbb{E}\left[\left|h_{l, l}\right|^{2}\right]$.

\section{SignAL MODEL}

Let there be $L_{1}$ interferers at the relay node and $L_{2}$ interferers at the destination. All the interfering channels are independent and identically distributed. The received signal at the destination can be expressed as [14],

$$
y_{D}=G h_{2} h_{1} x_{s}+G h_{2} \mathbf{h}_{I, 1} \mathbf{x}_{I, 1}^{T}+\mathbf{h}_{I, 2} \mathbf{x}_{I, 2}^{T}+G h_{2} n_{1}+n_{2}
$$

where $\mathbf{x}_{I, 1} \in C^{L_{1}}$ and $\mathbf{x}_{I, 2} \in C^{L_{2}}$ are the vectors with interference sources for the relay and receiver nodes respectively, and $\mathbf{h}_{I, 1} \in C^{L_{1}}$ and $\mathbf{h}_{I, 2} \in C^{L_{2}}$ are the corresponding fading channels from interferers to the relay and receiver nodes respectively. $G$ is the AF relay gain, $n_{1} \sim \mathcal{C N}\left(0, \sigma_{1}^{2}\right)$ and $n_{2} \sim \mathcal{C N}\left(0, \sigma_{2}^{2}\right)$ are AWGN at the relay and the destination respectively. Thus the SINR with arbitrary relay gain is given by

$$
\begin{array}{r}
\gamma_{\mathrm{SINR}}=\frac{G^{2}\left|h_{1}\right|^{2}\left|h_{2}\right|^{2} P_{S}}{G^{2}\left|h_{2}\right|^{2} \mathbf{h}_{I, 1} \boldsymbol{\Sigma}_{I, 1} \mathbf{h}_{I, 1}^{\dagger}+\mathbf{h}_{I, 2} \boldsymbol{\Sigma}_{I, 2} \mathbf{h}_{I, 2}^{\dagger}} \\
+G^{2}\left|h_{2}\right|^{2} \sigma_{1}^{2}+\sigma_{2}^{2}
\end{array}
$$

where $\boldsymbol{\Sigma}_{I, 1}=E\left\{\mathbf{x}_{I, 1}^{\dagger} \mathbf{x}_{I, 1}\right\}$ and $\boldsymbol{\Sigma}_{I, 2}=E\left\{\mathbf{x}_{I, 2}^{\dagger} \mathbf{x}_{I, 2}\right\}$ are diagonal matrices of the transmission powers of interfering signals at the relay node and the destination respectively and $P_{S}$ is the transmission power of the source node. Assuming that the network is interference limited, we set $\sigma_{i}^{2}=0$ in (3), where $i \in\{1,2\}$. With an ideal/hypothetical AF relay gain the end-to-end signal to interference power ratio (SIR) is given by [7],

$$
\gamma_{\mathrm{SIR}}=\frac{\gamma_{1} \gamma_{2}}{\gamma_{1} \gamma_{I, 2}+\gamma_{2} \gamma_{I, 1}}
$$

where $\gamma_{I, 1}$ and $\gamma_{I, 2}$ are the total instantaneous interference power at the relay and the destination respectively. Considering i.i.d. interferers both at the relay and destination, the probability density function (PDF) of the end-to-end signal to interference ratio (SIR) $\gamma_{\mathrm{SIR}}$ at the destination can be written as [7],

$$
\begin{aligned}
& f_{\gamma_{\mathrm{SIR}}}(\gamma)=\frac{L_{1} L_{2} \Gamma\left(L_{1}\right) \Gamma\left(L_{2}\right)}{\Gamma\left(L_{1}+L_{2}+1\right)}\left(1+\frac{\lambda_{I, 1} \gamma}{\lambda_{1}}\right)^{-L_{1}}\left(1+\frac{\lambda_{I, 2} \gamma}{\lambda_{2}}\right)^{-L_{2}} \\
& \times\left[\left(\frac{L_{1} \lambda_{I, 1}}{\lambda_{1}+\lambda_{I, 1} \gamma}+\frac{L_{2} \lambda_{I, 2}}{\lambda_{2}+\lambda_{I, 2} \gamma}\right){ }_{2} F_{1}\left(L_{1}, L_{2} ; L_{1}+L_{2}+1 ; k_{1}(\gamma)\right)\right. \\
& \left.+\frac{L_{1} L_{2} \lambda_{I, 1} \lambda_{I, 2} k_{2}(\gamma) \gamma}{\left(L_{1}+L_{2}+1\right) \lambda_{1} \lambda_{2}}{ }_{2} F_{1}\left(L_{1}+1, L_{2}+1 ; L_{1}+L_{2}+2 ; k_{1}(\gamma)\right)\right]
\end{aligned}
$$

where ${ }_{2} F_{1}(a, b ; c ; z)$ is Gauss hypergeometric function defined as [16, eq. (15.1.1)]. $\lambda_{I, 1}$ and $\lambda_{I, 2}$ are the average power of an interferer at the relay and the destination respectively, and $k_{\nu}(\gamma)=\frac{\nu+\frac{\lambda_{I, 1} \gamma}{\lambda_{1}}+\frac{\lambda_{I, 2} \gamma}{\lambda_{2}}}{\left(1+\frac{\lambda_{I, 1} \gamma}{\lambda_{1}}\right)^{\nu}\left(1+\frac{\lambda_{I, 2} \gamma}{\lambda_{2}}\right)^{\nu}}$.

\section{ERROR EXPONENT: I.I.D. INTERFERENCE NETWORK}

The random coding error exponent is defined as a function of input distribution function $Q(x)$, a factor $\rho \in[0,1]$ and rate $R \leq C$ (for details please read ch. 5 of [9]), which is jointly optimized over $Q(x)$ and $\rho$ at a desired rate $R$. However, the Gaussian input distribution has often been used in many publications such as in [12], [17] to avoid the mathematical complexity involved in the joint optimization of the reliability function. This assumption provides near optimal result for the error exponent at a rate near the channel capacity. We analyze the error exponent for i.i.d. interference network where all the interfering channels to the relay and to the destination are

\footnotetext{
${ }^{1}$ Hypothetical relay gain proposed by Hasna et.al. in [15] simply inverses the instantaneous channel gain as $G^{2}=\frac{P_{R}}{P_{S}\left|h_{1}\right|^{2}}$,
} 
independent and identically distributed. The error exponent of the dual hop AF network with Gaussian input distribution can be written as [9],

$$
E_{r}(R)=\max _{0 \leq \rho \leq 1}\left\{E_{0}(\rho)-2 \rho R\right\}
$$

with

$$
E_{0}(\rho)=-\ln \mathbb{E}_{\gamma_{\mathrm{SIR}}}\left\{\left(1+\frac{\gamma}{1+\rho}\right)^{-\rho}\right\}
$$

$\mathbb{E}_{\gamma_{\mathrm{SIR}}}(\gamma)$ denotes the statistical expectation operation over random variable $\gamma_{\mathrm{SIR}}$. Using the series expression of Gauss hypergeometric function as in [16, eq. (15.1.1)] in eq. (5) and (7), random coding error exponent over i.i.d. interference channels can be written as,

$$
\begin{aligned}
& E_{0}(\rho)=-\ln \left[L_{1} L_{2} \sum_{n=0}^{\infty} \sum_{k=0}^{n}\left(\begin{array}{l}
n \\
k
\end{array}\right)\left(\frac{\lambda_{I}}{\lambda}\right)^{k+1}\right. \\
& \times \frac{\Gamma\left(L_{1}+n\right) \Gamma\left(L_{2}+n\right) 2^{k}}{\Gamma(\rho) \Gamma\left(L_{1}+L_{2}+n+1\right) n !}\left\{\frac{\left(L_{1}+L_{2}\right)}{\Gamma\left(L_{1}+L_{2}+2 n+1\right)}\right. \\
& \times \int_{0}^{\infty} \gamma^{k} G_{1,1}^{1,1}\left(\frac{\lambda_{I} \gamma}{\lambda} \mid \begin{array}{c}
-L_{1}-L_{2}-2 n \\
0
\end{array}\right) G_{1,1}^{1,1}\left(\frac{\gamma}{1+\rho} \mid \begin{array}{c}
1-\rho \\
0
\end{array}\right) d \gamma \\
& +\frac{2 \lambda_{I}}{\lambda} \frac{\left(L_{1}+n\right)\left(L_{2}+n\right)}{\Gamma\left(L_{1}+L_{2}+2 n+3\right)\left(L_{1}+L_{2}+n+1\right)} \\
& \times \int_{0}^{\infty} \gamma^{k+1} G_{1,1}^{1,1}\left(\frac{\lambda_{I} \gamma}{\lambda} \mid \begin{array}{c}
\left.-L_{1}-L_{2}-2 n-3\right) \\
0
\end{array}\right) \\
& \left.\left.\times G_{1,1}^{1,1}\left(\frac{\gamma}{1+\rho} \mid \begin{array}{c}
1-\rho \\
0
\end{array}\right) d \gamma\right\}\right]
\end{aligned}
$$

Using [18, eq. (8.24.1.1)] and after some manipulations the RCEE can be expressed as (9) shown at the bottom of the page 4 , where $K$ represents the maximum number of sum terms required for convergence. From numerical calculations we found $K \cong 100000$ is sufficient for convergence. $G_{p, q}^{m, n}\left(z \mid \begin{array}{l}a_{p} \\ b_{q}\end{array}\right)$ is the Meijer-G function defined as [18, eq. 8.2.1.1] and $\alpha=\frac{\lambda}{\lambda_{I}}$.

\section{ERGODIC CAPACITY}

Ergodic capacity $\langle C\rangle$ of this dual hop network is given by,

$$
\begin{aligned}
\langle C\rangle & =\frac{1}{2}\left[\frac{\partial E_{0}(\rho)}{\partial \rho}\right]_{\rho=0} \\
& =\frac{1}{2} \int_{0}^{\infty} \ln (1+\gamma) f_{\gamma_{\mathrm{SIR}}}(\gamma) d \gamma
\end{aligned}
$$

Let

$$
\mathcal{J}(\gamma, \alpha, k)=\int_{0}^{\infty} \gamma^{k} \ln (1+\gamma)\left(1+\frac{\gamma}{\alpha}\right)^{-n} d \gamma
$$

Using [19, eq. 2.6.10.60], for $\alpha>1$ we have,

$$
\begin{aligned}
\mathcal{J}(\gamma, \alpha, k) & =\alpha^{k+1} \mathbf{B}(k+1, n-k-1)\left[\frac{1}{\alpha^{k+1}} \sum_{l=0}^{\infty} \frac{(k+1)_{l}}{l !}\right. \\
& \left.\times\left(1-\frac{1}{\alpha}\right)^{l} \psi(n+l)-\psi(n-k-1)\right]
\end{aligned}
$$

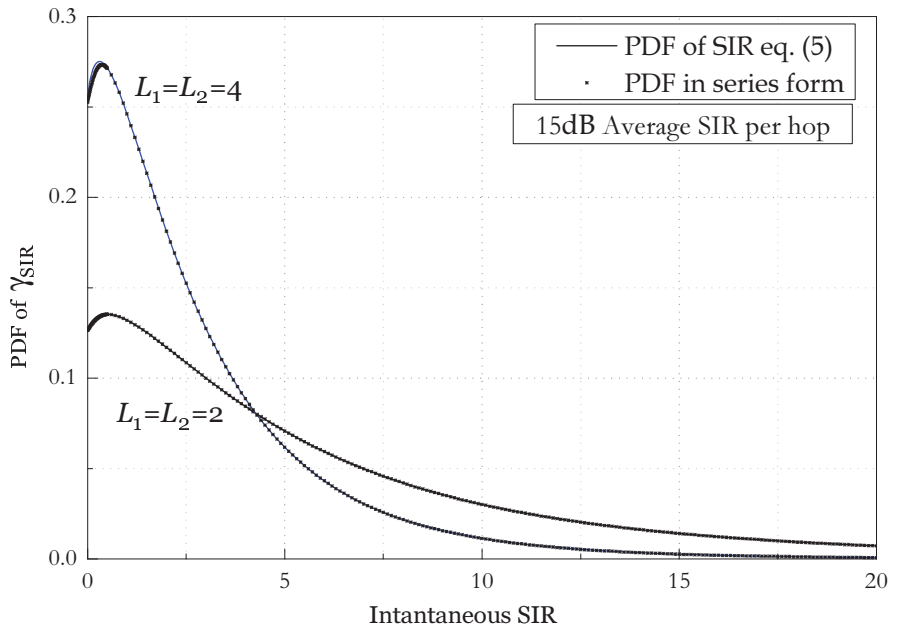

Fig. 2. PDF of destination SIR $f_{\gamma_{\mathrm{SIR}}}(\gamma)$ as a function of instantaneous SIR.

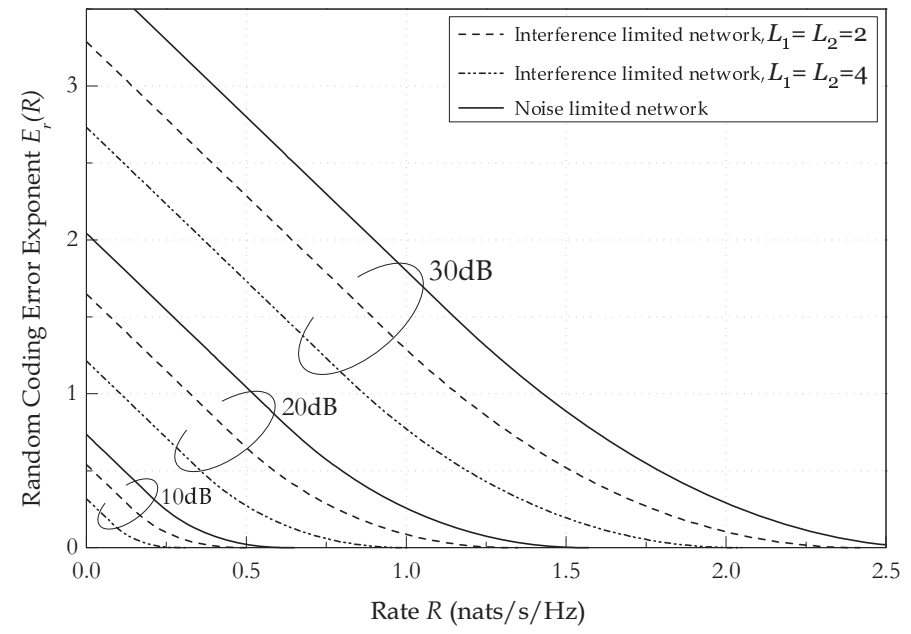

Fig. 3. Random coding error exponent as a function of rate $R$ in nats/s/Hz for different SIRs per hop.

where $\mathbf{B}(a, b)$ is Beta function, $(a)_{n}$ is the Pochhammer symbol and $\psi(z)$ is the Euler Psi function. Using the above solution the ergodic capacity $\langle C\rangle$ of the interference limited cooperative relay network can be written as (13), shown at the bottom of the page 4 .

\section{NUMERICAL RESUlts}

For numerical evaluation we assume the average gain of the main channels and the interfering channels are unity. Furthermore, in noise limited networks we consider the noise variances at the relay and the destination are equal to $\sigma^{2}$. RCEE and the ergodic capacity expressions (9) and (13) contain functions of infinite sums. We observed that for $n \geq 100000$ sum terms, the expressions converge to the simulation results. In all figures, parameters average SIR per hop in interference limited network and average SNR per hop when the network is noise limited have been used.

Fig. 2 compares the probability density function in equation 


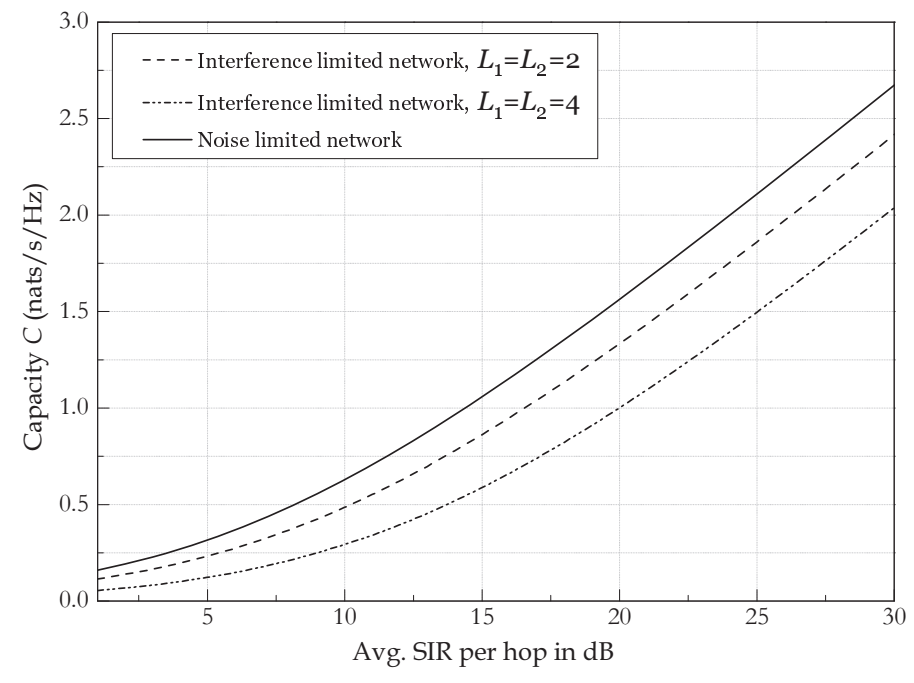

Fig. 4. Ergodic capacity as a function of SIR per hop for different number of interferers.

(5) with the series representation of Gauss hypergeometric function as given in [16, eq. (15.1.1)]. The figure shows a perfect match of the PDF plots when $n>100000$, and thus verifies the implementation issue of the series expression of Hypergeometric function in this analysis using a limited number of sum terms.

Fig. 3 plots the random coding error exponent as a function of data rate in nats/s/Hz for 10,20 , and $30 \mathrm{~dB}$ average SIR per hop. The figure compares the RCEE of interference limited network consisting of 2 and 4 interferers at the relay and destination nodes with the noise limited network. It shows that, noise limited networks outperform the interference limited networks even when there are only 2 interferers both at the relay and destination. At $20 \mathrm{~dB}$ per hop average SIR (SNR in noise limited network), for example, the RCEE of noise limited network is almost 1.6 times higher than the interference limited networks with 2 interferers both at the relay and the destination.

Fig. 4 compares the capacity of the interference limited and noise limited networks as a function of per hop average SIR and SNR in Rayleigh fading channels. The figure shows that noise limited networks perform quite better compared to interference limited networks even when only 2 interferers exists both at the relay and the destination. And, when the number of interferer increases the network suffers by significant amount of reduction in its channel capacity. For example with $L_{1}=L_{2}=4$ channel capacity of the network is reduced by $36 \%$ compared to the noise limited network.

\section{CONCLUSION}

In this paper, we have derived the RCEE of cooperative relay network in presence of arbitrary number of i.i.d. interferers using an ideal gain AF relay. The expression of ergodic capacity of the network is also derived. Numerical results on RCEE and ergodic capacity show that interference limited networks perform worse than noise limited networks even when only a very few interferers exist in the network.

\section{ACKNOWLEDGMENTS}

This research was supported by University of Wollongong research project "Souring Eagle" and Australian Research Council (ARC) discovery research grant No. DP0879507.

$$
\begin{aligned}
E_{0}(\rho)= & -\ln \left[L_{1} L_{2} \sum_{n=0}^{K} \sum_{k=0}^{n}\left(\begin{array}{l}
n \\
k
\end{array}\right) \frac{\Gamma\left(L_{1}+n\right) \Gamma\left(L_{2}+n\right) 2^{k}}{\Gamma(\rho) \Gamma\left(L_{1}+L_{2}+n+1\right) n !}\right. \\
& \times\left\{\frac{\left(L_{1}+L_{2}\right)}{\Gamma\left(L_{1}+L_{2}+2 n+1\right)} G_{2,2}^{2,2}\left(\frac{\alpha}{1+\rho} \mid \begin{array}{c}
1-\rho,-k \\
0, L_{1}+L_{2}+2 n-k-1
\end{array}\right)\right. \\
+ & \left.\left.\frac{2\left(L_{1}+n\right)\left(L_{2}+n\right)}{\Gamma\left(L_{1}+L_{2}+2 n+3\right)\left(L_{1}+L_{2}+n+1\right)} G_{2,2}^{2,2}\left(\frac{\alpha}{1+\rho} \mid \begin{array}{c}
1-\rho,-k-1 \\
0, L_{1}+L_{2}+2 n-k
\end{array}\right)\right\}\right]
\end{aligned}
$$

$$
\begin{aligned}
\langle C\rangle & =\sum_{n=0}^{K} \sum_{k=0}^{n}\left(\begin{array}{l}
n \\
k
\end{array}\right) \frac{L_{1} L_{2} \Gamma\left(L_{1}+n\right) \Gamma\left(L_{2}+n\right) 2^{k-1}}{\Gamma\left(L_{1}+L_{2}+n+1\right) n !}\left[\left(L_{1}+L_{2}\right) \mathbf{B}\left(k+1, L_{1}+L_{2}+2 n-k\right)\right. \\
& \left\{\left\{\sum_{l=0}^{\infty} \frac{(k+1)_{l}}{\alpha^{k+1} l !}\left(1-\frac{1}{\alpha}\right)^{l} \psi\left(L_{1}+L_{2}+2 n+l+1\right)-\psi\left(L_{1}+L_{2}+2 n-k\right)\right\}\right. \\
& +\frac{2\left(L_{1}+n\right)\left(L_{2}+n\right)}{\left(L_{1}+L_{2}+n+1\right)} \mathbf{B}\left(k+2, L_{1}+L_{2}+2 n-k+1\right)\left\{\sum_{l=0}^{\infty} \frac{(k+2)_{l}}{\alpha^{k+2} l !}\left(1-\frac{1}{\alpha}\right)^{l}\right. \\
& \left.\left.\times \psi\left(L_{1}+L_{2}+2 n+l+3\right)-\psi\left(L_{1}+L_{2}+2 n-k+1\right)\right\}\right],
\end{aligned}
$$




\section{REFERENCES}

[1] T. M. Cover and A. A. El Gamal, "Capacity theorems for the relay channel," IEEE Transactions on Information Theory, vol. 25, no. 5, pp. 572-584, Sep. 1979.

[2] G. Kramer, M. Gastpar, and P. Gupta, "Cooperative strategies and capacity theorems for relay networks," IEEE Transactions on Information Theory, vol. 51, no. 9, pp. 3037-3063, Sept 2005.

[3] S. Mohajer, S. N. Diggavi, C. Fragouli, and D. N. C. Tse, "Approximate capacity of a class of gaussian interference-relay networks," IEEE Transactions on Information Theory, vol. 57, no. 5, pp. 2837-2864, May 2011.

[4] H. A. Suraweera, H. K. Garg, and A. Nallanathan, "Performance analysis of two hop amplify-and-forward systems with interference at the relay," IEEE Communications Letters, vol. 14, no. 8, pp. 692 -694, Aug. 2010

[5] F. S. Al-Qahtani, T. Q. Duong, C. Zhong, K. A. Qaraqe, and H. Alnuweiri, "Performance analysis of dual-hop af systems with interference in nakagami-m fading channels," IEEE Signal Processing Letters, vol. 18 , no. 8 , pp. $454-457$, Aug. 2011.

[6] W. Xu, J. Zhang, and P. Zhang, "Outage probability of two-hop fixed-gain relay with interference at the relay and destination," IEEE Communications Letters, vol. 15, no. 6, pp. 608 -610, Jun. 2011.

[7] D. Lee and J. H. Lee, "Outage probability for dual-hop relaying systems with multiple interferers over rayleigh fading channels," IEEE Transactions on Vehicular Technology, vol. 60, no. 1, pp. 333 -338, Jan. 2011.

[8] C. E. Shannon, "Probability of error for optimal codes in a gaussian channel," Bell System Technical Journal, vol. 38, p. 611656, Sept. 1959.

[9] R. G. Gallager, Information Theory and Reliable Communication. New York: Wiley, 1968

[10] L. Weng, S. S. Pradhan, and A. Anastasopoulos, "Error exponent regions for gaussian broadcast and multiple-access channels," IEEE Transactions on Information Theory, vol. 54, no. 7, pp. 2919-2942, 2008.
[11] H. Q. Ngo, T. Quek, and H. Shin, "Random coding error exponent for dual-hop nakagami-m fading channels with amplify-and-forward relaying," IEEE Communications Letters, vol. 13, no. 11, pp. 823-825, Nov. 2009.

[12] H. Q. Ngo, T. Q. S. Quek, and H. Shin, "Amplify-and-forward twoway relay networks: Error exponents and resource allocation," IEEE Transactions on Communications, vol. 58, no. 9, pp. 2653-2666, Sept. 2010.

[13] B. Barua, M. Abolhasan, F. Safaei, and D. R. Franklin, "On the error exponent of amplify and forward relay networks," IEEE Communications Letters, vol. 15, no. 10, pp. 1047-1049, Oct. 2011

[14] B. Barua, M. Abolhasan, D. Franklin, and F. Safaei, "Outage probability analysis of dual hop relay networks in presence of interference," 2012, submitted for publication. [Online]. Available: http://arxiv.org/abs/1210.4243

[15] M. O. Hasna and M.-S. Alouini, "End-to-end performance of transmission systems with relays over rayleigh-fading channels," IEEE Transactions on Wireless Communications, vol. 2, no. 6, pp. 1126-1131, Nov. 2003.

[16] M. Abramowitz and I. A. Stegun, Handbook of Mathematical Functions With Formulas, Graphs, and Mathematical Tables. New York: Dover, 1972.

[17] T. Ericson, "A Gaussian channel with slow fading," IEEE Transactions on Information Theory, vol. 16, no. 3, pp. 353-355, May 1970.

[18] A. P. Prudnikov, Y. A. Brychkov, and O. I. Marichev, Integrals and Series: More Special Functions. New York: Gordon and Breach Science, 1990, vol. 3.

[19] _ Integrals and Series: Elementary Functions. New York: Gordon and Breach Science, 1991, vol. 1. 